\title{
Preliminary pathogenicity screening of Verticillium spp. on kiwifruit in New Zealand
}

\author{
Kieran D. Mellow ${ }^{1}$, Joy L. Tyson ${ }^{1}$, Michael A. Manning ${ }^{1}$ and Peter J. Wright ${ }^{2}$ \\ ${ }^{1}$ The New Zealand Institute for Plant and Food Research Limited, Private Bag 92169, Auckland, \\ 1142, New Zealand \\ ${ }^{2}$ The New Zealand Institute for Plant and Food Research Limited, Cronin Rd, Pukekohe, 2676, \\ New Zealand \\ ${ }^{*}$ Corresponding author: Kieran.Mellow@plantandfood.co.nz
}

\begin{abstract}
Plant-pathogenic Verticillium species have been present in New Zealand for many years, and have been considered minor wilt pathogens of kiwifruit. However, an outbreak of Verticillium nonalfalfae (previously identified as Verticillium alboatrum) causing wilt and death of the kiwifruit cultivar Actinidia chinensis var. chinensis 'Hort16A' in Chile has raised questions around the pathogenicity and significance of New Zealand Verticillium species. This study investigated the pathogenicity of New Zealand isolates of Verticillium spp. to 'Hort16A'. Three isolates of Verticillium dahliae and one of V. alboatrum sensu stricto, previously recovered from kiwifruit in New Zealand, were tested for pathogenicity against 'Hort16A' by artificial inoculation of young vines. Disease assessments were carried out monthly. Symptoms observed ranged from minor wilt to vine death. The V. alboatrum isolate appeared the most aggressive. Although there is evidence of some pathogenicity on kiwifruit within this group of isolates from Verticillium species in New Zealand, they appear less aggressive than those recorded in Chile. However, this cannot be confirmed without testing isolates from both countries concurrently under the same conditions.
\end{abstract}

Keywords Verticillium dahliae, Verticillium alboatrum, Verticillium nonalfalfae, Actinidia chinensis

\section{INTRODUCTION}

The genus Verticillium comprises a group of plant-pathogenic fungi that are of economic importance to a variety of agricultural crops in many parts of the world. The soil-borne nature of Verticillium spp. makes control of diseases caused by these pathogens problematic. Some Verticillium species, such as $V$. dahliae, produce long-lived microsclerotia and are able to survive in the soil for more than a decade, adding to the difficulty of control of verticillium wilts (Daayf 2015).

In 2005, a new disease was seen causing sudden wilt, dieback and death of gold-fleshed kiwifruit (Actinidia chinensis var. chinensis 'Hort16A') in Chile. The Chilean outbreak of verticillium wilt was severe, with losses of up to $80 \%$ being reported in some orchards (Auger et al. 2009).
The symptoms were consistent with descriptions of vascular infection by Verticillium spp. in other plant species (Manning unpublished data). The causal pathogen was isolated and identified as Verticillium alboatrum (Fullerton \& Young unpublished $^{\mathrm{a}}$ ).

In 2014, $V$. alboatrum was taxonomically revised and split into three species ( $V$. alboatrum sensu stricto, $V$. alfalfae and $V$. nonalfalfae) (Inderbitzin et al. 2011). The isolates of $V$. alboatrum from Actinidia in Chile have subsequently been re-classified as $V$. nonalfalfae (Kasson et al. 2014). Molecular characterisation of $V$. nonalfalfae isolates associated with lethal wilt of Actinidia chinensis in Chile by Kasson et al. (2014), placed them in a distinct intra-specific group designated multilocus sequence type 2 (MLST 2). Although $V$. nonalfalfae has been 
found in New Zealand, MLST 2 has not been recorded (P. Johnston, Landcare Research, pers. comm.).

Verticillium wilt of kiwifruit caused by Verticillium dahliae was first recorded in New Zealand on Actinidia chinensis var. deliciosa 'Hayward' in 1982. The primary symptom was cane dieback and associated wood discoloration (Hill 1982). At this time, it was described as a minor disease (Brook 1990). Manning et al. (unpublished $^{\mathrm{b}}$ ) reported Verticillium dahliae causing spongy bark disease of 'Hort16A'; it was also associated with vine death of kiwifruit in Paengaroa and Whakatane, New Zealand. However, the significance of Verticillium spp. to the New Zealand kiwifruit industry is largely unknown.

This study aimed to test New Zealand isolates of Verticillium spp. for pathogenicity to kiwifruit, using young vines of 'Hort16A', as this cultivar was identified as the most susceptible in Chile (Fullerton et al. unpublished ${ }^{\mathrm{c}}$ ). This study was conducted after the New Zealand industry stopped growing 'Hort16A' (due to its susceptibility towards Pseudomonas syringae pv. actinidiae (Psa)) but the results are still relevant to the current kiwifruit industry in New Zealand.

\section{MATERIALS AND METHODS}

\section{Plant material}

One hundred 'Hort16A' plants were used in this study. All plants were grown from tissue-cultured plantlets obtained from Multiflora Laboratories, Auckland, New Zealand. The plantlets were potted into $8-\mathrm{cm}$ diameter polythene planting bags and grown in glasshouse conditions at
The New Zealand Institute for Plant and Food Research Limited (PFR), Mount Albert Research Centre (average temperature of $25 \pm 5^{\circ} \mathrm{C}$ ).

The plants were moved to the PFR Pukekohe Research Station 6-9 months post-tissue culture and transferred to $12 \mathrm{~cm}$ diameter potting bags containing sterile potting mix with slow-release fertiliser and were irrigated via drippers. The plants remained there for the duration of the trial, tied to an overhead wire and maintained at $2 \mathrm{~m}$ in height by pruning.

\section{Verticillium isolates}

New Zealand isolates of Verticillium spp. were obtained from collections held by PFR (Table 1). All the isolates of Verticillium spp. used in this study were from kiwifruit (Actinidia species) and identified using standard morphological techniques.

Prior to inoculation of the 'Hort16A' vines, the isolates from each Verticillium species were grown on Oxoid malt extract agar (MEA) at room temperature for 7 days.

Representative isolates have been deposited into the Manaaki Whenua - Landcare Research International Collection of Microorganisms from Plants (ICMP) as ICMP 22720 (V. alboatrum, cc164) and ICMP 22666 (V. dahliae, MM765).

\section{Inoculation}

All vines were inoculated on 11 July 2017. Half of the vines were in an active growth phase, while half had entered dormancy. Ten vines of 'Hort16A' of each growth stage were inoculated with each treatment (four isolates of Verticillium spp. and a non-inoculated control).

Table 1 New Zealand isolates of Verticillium spp. used for pathogenicity testing. All isolates originate from kiwifruit (Actinidia spp.).

\begin{tabular}{lllll}
\hline Verticillium sp. & Isolate ID & Collection date & Location & Isolated from \\
\hline V. alboatrum $^{*}$ & cc164 & May 2003 & No. 1 Road, Te Puke & Leaf \\
V. dahliae & MM765 & Feb 2009 & Old Coach Rd, Te Puke & Leader \\
V. dahliae & MM766 & Feb 2009 & Old Coach Rd, Te Puke & Trunk, $0.2 \mathrm{~m}$ \\
V. dahliae & MM768 & Feb 2009 & Old Coach Rd, Te Puke & Trunk, $0.8 \mathrm{~m}$ \\
\hline
\end{tabular}

${ }^{\star}$ The $V$. alboatrum isolate used in this study has since been identified using molecular techniques as V. alboatrum sensu stricto (P. Johnston, Landcare Research, pers. comm.) 
An approximately 3-mm deep, downwardslanting cut was made on the main stem from the outer bark to the inner wood with a sterile scalpel, $5 \mathrm{~cm}$ above the soil. A 5-mm mycelial plug of inoculum was placed into the wound using a sterile scalpel and the inoculation site was wrapped with Parafilm ${ }^{\circledR}$ to allow local mycelial infection of vines by the various isolates of Verticillium species. The Parafilm was removed $48 \mathrm{~h}$ after inoculation. Plugs of sterile malt agar were used for all control vines.

\section{Assessment and re-isolation}

Assessments of the vines were made monthly. At each assessment date, any symptoms of disease such as dieback and wilt were recorded. At any stage of the trial, when a vine was found to exhibit severe symptoms (severe wilt, near total dieback, or death), isolations were performed to identify the cause of the symptoms. All remaining vines were harvested 9 months after inoculation. After harvest, the lower stem of each vine encompassing the inoculation point was split vertically and the extent of stem discoloration above and below the inoculation point measured.

To establish whether the inoculated Verticillium spp. remained alive in the vine tissues, the stems were surface sterilised and small pieces of tissue from the inoculation point were placed onto Difco potato dextrose agar (PDA) amended with antibiotics (ampicillin and streptomycin). In addition, a short piece of stem (approximately $20 \mathrm{~cm}$ ), including the inoculation point, was incubated at high humidity for 7 days, then examined for the formation of Verticillium spp. conidiophores on the cut surfaces. For vines that died, re-isolation was attempted at the inoculation site and at 1,2 and $5 \mathrm{~cm}$ above the inoculation site and at 1 and $2 \mathrm{~cm}$ below the inoculation site, and from the roots.

\section{RESULTS}

A wide range of symptoms was observed during the experiment, from a minor wilt to death of the vine. The overall results are summarised in Figure 1. Results of stem inoculations from specific treatments are summarised in Figure 2. At no time did any of the non-inoculated control vines develop symptoms of verticillium wilt. Isolate cc164 (V. alboatrum sensu stricto) appeared to be more aggressive than the three $V$. dahliae isolates used in this study. All ten vines inoculated with

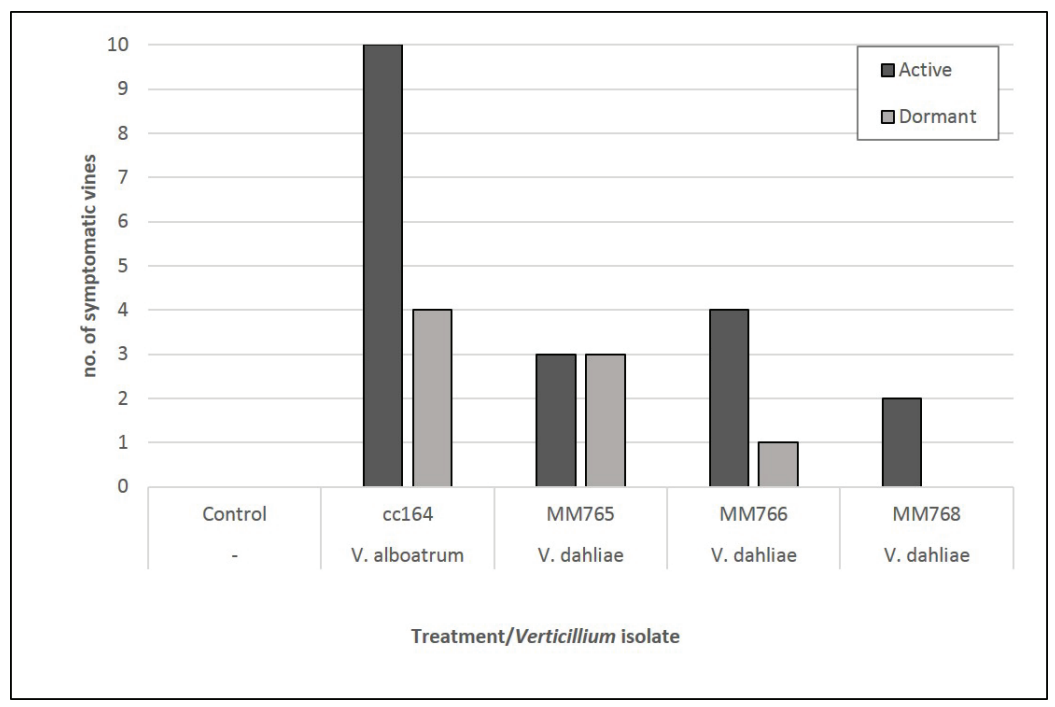

Figure 1 Number of active and dormant Actinidia chinensis var. chinensis 'Hort16A' plants that developed verticillium wilt symptoms in each treatment within 9 months of inoculation with $V$. alboatrum or $V$. dahliae $(n=10)$. 


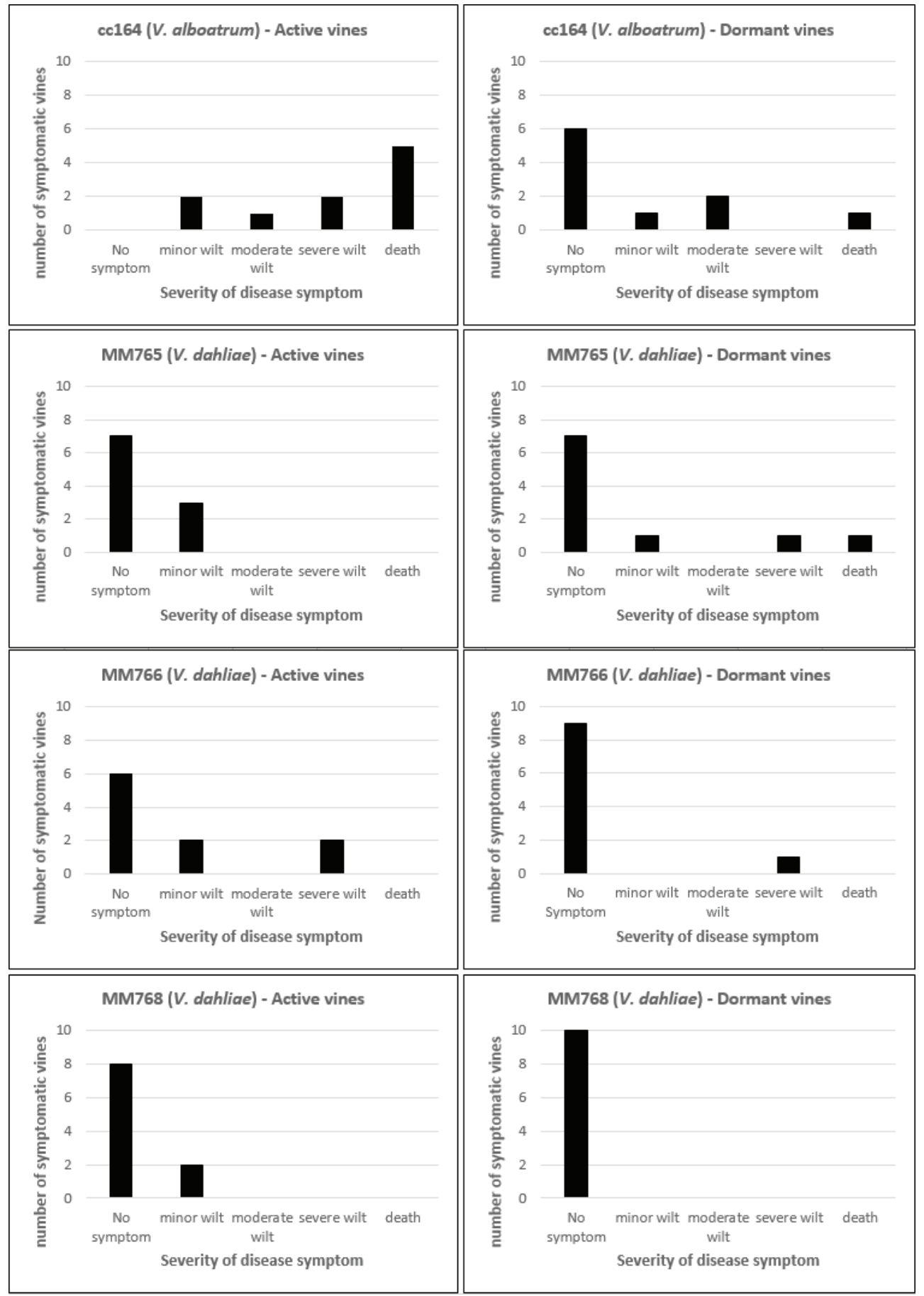

Figure 2 Number of Actinidia chinensis var. chinensis 'Hort16A' plants that developed verticillium wilt symptoms of different severities in each treatment within 9 months of inoculation with $V$. alboatrum or $V$. dahliae $(\mathrm{n}=10)$. 
isolate cc164 during active growth developed symptoms, with five dying. Conversely, of the vines inoculated with the three $V$. dahliae isolates, only one vine died (Fig. 2).

Many of the vines that developed symptoms of verticillium wilt recovered, particularly those inoculated with $V$. dahliae. Some of these recovering vines also developed vigorous regrowth at the crown below the inoculation site.

Verticillium species were recovered from 23 of the 27 vines ( $82 \%)$ that developed symptoms. Of the vines that died $(n=7)$, isolates of Verticillium species were recovered up to $5 \mathrm{~cm}$ above the inoculation site of 5 of the 7 vines. Isolates of Verticillium species were recovered from the roots of only one vine during the experimental time frame ( 9 months). Of the 53 inoculated vines that remained asymptomatic throughout the duration of the experiment, Verticillium species were re-isolated from 25 (47\%) of these.

\section{DISCUSSION}

The results from this study give an indication of the pathogenicity of Verticillium alboatrum sensu stricto and $V$. dahliae from 'Hort16A' kiwifruit in New Zealand. Also, they are consistent with observations of verticillium wilt by Manning et al. (unpublished ${ }^{\mathrm{b}}$ ) in orchards at Opotiki and Paengaroa, where relatively few vine deaths were observed despite there being large numbers of symptomatic vines from which $V$. dahliae was isolated. Although all of the isolates of Verticillium spp. tested in this study were pathogenic to some extent on 'Hort16A' kiwifruit, the isolates appeared to be less aggressive than those recorded in Chile.

A similar study in Chile on 'Hort16A' and 'Hayward' using Chilean isolates of $V$. nonalfalfae reported that $100 \%$ of inoculated vines developed typical wilt and dieback symptoms 8 weeks after inoculation. Eleven months after inoculation, budding of 'Hort16A' was completely inhibited while 'Hayward' budding was unaffected (Auger et al. 2011). However, 'Hayward' is tolerant in the field in Chile. The findings of this study, in conjunction with the observed resistance of Chilean 'Hayward' orchards, suggests that invasive inoculation tests are not necessarily representative of symptoms observed in an orchard environment.

Of the four Verticillium isolates tested in this study, the isolate identified as V. alboatrum showed the greatest pathogenicity. Similar studies in Chile testing for pathogenicity of Verticillium spp. using local strains of $V$. nonalfalfae found high pathogenicity to the 'Hort16A' cultivar (Auger et al. 2011). The 'Hayward' cultivar is relatively tolerant to the disease, and 'Hayward' orchards do not appear affected by $V$. nonalfalfae in Chile (Auger et al. 2011). Investigating the pathogenicity of New Zealand Verticillium spp. with other cultivars of kiwifruit would give more insight into the potential threat these pathogens have on the kiwifruit industry in New Zealand.

Despite the presence of $V$. nonalfalfae in New Zealand, the highly pathogenic MLST 2 group of $V$. nonalfalfae identified in Chile has not been recorded in New Zealand. Furthermore, there have been no records of $V$. nonalfalfae on kiwifruit in New Zealand. Research investigating the potential threat of New Zealand species of Verticillium to a variety of host crops is ongoing (P. Johnston, Landcare Research, pers. comm.). Following the taxonomic changes within the Verticillium genus, there is limited knowledge of the host range of $V$. alboatrum. The only two isolates reported as $V$. alboatrum sensu stricto were from potato (Inderbitzin et al. 2011).

The results from pathogenicity screening suggest that the tested isolate of $V$. alboatrum has greater pathogenicity towards 'Hort16A' than $V$. dahliae. Interestingly, the isolate of $V$. alboatrum used in this study was originally collected from the surface of a leaf from a healthy kiwifruit vine, and there are no records of kiwifruit wilt in orchards caused by this species. Despite the pathogenicity of the $V$. dahliae isolates from this study appearing low, it has been associated with multiple, although sporadic, occurrences of problematic wilt of kiwifruit in New Zealand (Hill 1982; Manning et al. unpublished ${ }^{\mathrm{b}}$ ). However, the biggest threat of verticillium wilts in New Zealand remains the risk of introduction of Verticillium nonalfalfae MLST 2. 
Future work for better understanding the potential threat of $\mathrm{New}$ Zealand isolates of Verticillium spp. could include investigating any genetic similarities and differences between the New Zealand and Chilean isolates using the protocols of Kasson (2014). Testing of the Chilean and New Zealand Verticillium species concurrently would give greater confidence in the differences in pathogenicity between the species. Ensuring the prevention of the introduction of highly pathogenic strains of Verticillium species (such as the $V$. nonalfalfae MLST 2 group from Actinidia sp. in Chile) into New Zealand is essential to protecting the kiwifruit industry from verticillium wilts.

\section{REFERENCES}

Auger J, Perez I, Fullerton RA, Esterio M 2009. First report of Verticillium wilt of gold kiwifruit, Actinidia chinensis cv. Hort16A, caused by Verticillium albo-atrum in Chile. Plant Disease 93: 553-553.

Auger J, Pérez I, Esterio M, Fullerton RA 2011. Outbreak of a lethal wilt form of gold kiwifruit, Actinidia chinensis 'Hort16A' in Chile. Acta Horticulturae 913: 473-479.

Brook PJ 1990. Diseases of kiwifruit. In: Warrington IJ, Weston GC Ed. Kiwifruit science and management. Auckland, New Zealand, New Zealand Society for Horticultural Science. Pp. 420-428.

Daayf F 2015. Verticillium wilts in crop plants: Pathogen invasion and host defence responses. Canadian Journal of Plant Pathology 37: 8-20.

Hill CF 1982. New plant disease records in New Zealand, 1974-1982. New Zealand Journal of Botany 20: 355-359.

Inderbitzin P, Bostock RM, Davis RM, Usami T, Platt HW 2011. Phylogenetics and taxonomy of the fungal vascular wilt pathogen Verticillium, with the descriptions of five new species. PLoS ONE 6: 1-22.

Kasson MT, Short DP, O’Neal ES, Subbarao KV, Davis DD 2014. Comparative pathogenicity, biocontrol efficacy, and multilocus sequence typing of Verticillium nonalfalfae from the invasive Ailanthus altissima and other hosts. Phytopathology 104: 282-292.

\section{ENDNOTES}

a Fullerton RA, Young WB 2009 (unpublished). Report of a visit to Chile to identify options for technical support for growers of 'Hort 16A' kiwifruit. Plant \& Food Research, SPTS No. 2719. Auckland, New Zealand. 13 p.

b Manning MA, Casonato SG, Currie M, Weber P 2010 (unpublished). Reducing the threat from New Zealand vine diseases. Plant \& Food Research, SPTS No. 3721. Auckland, New Zealand. 46 p.

c Fullerton RA, Duboy F, Callejas MJ, Martin RA, Auger J, Pérez I 2008 (unpublished). Report on a visit to Chile to further investigate Verticillium disease of 'Hort16A'. HortResearch Client Report No. 25516. Auckland, New Zealand. 55 p. 